\title{
Mesh erosion after vertical-banded gastroplasty
}
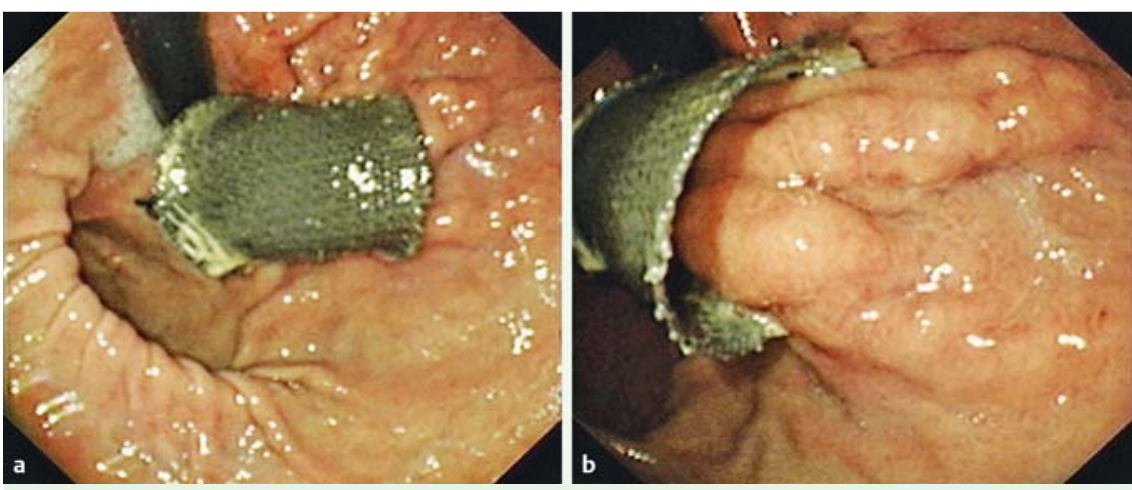

Fig. 1 a, b In a 33-year-old man with a vertical-banded gastroplasty, esophagogastroduodenoscopy revealed part of the mesh eroding into the stomach 10 years after the primary surgery.

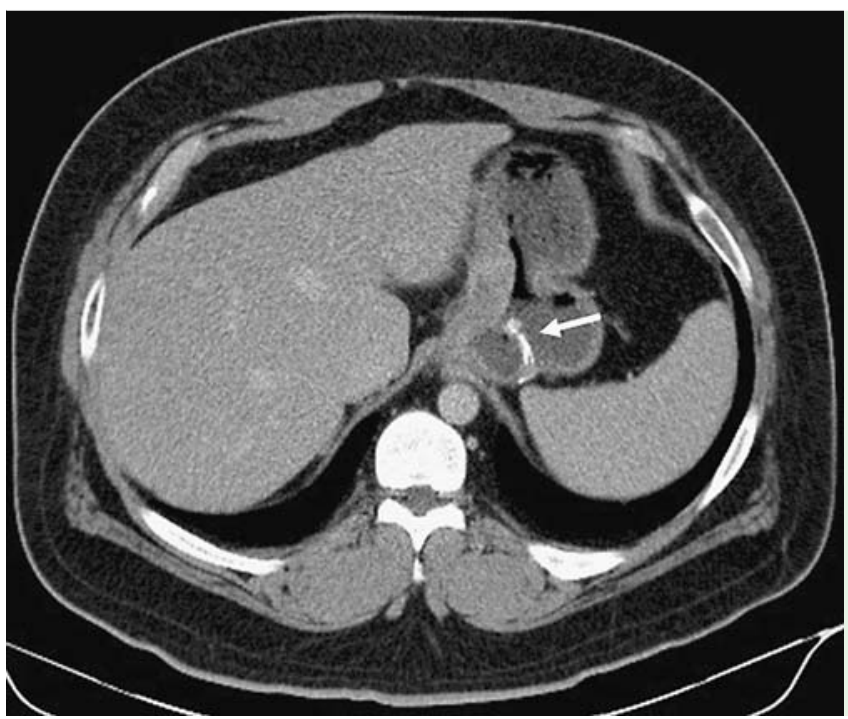

Fig. 2 Abdominal computed tomography revealed interruption of the mesh with intraluminal protrusion (arrow).

A 33-year-old man underwent verticalbanded gastroplasty (VBG) for the management of morbid obesity 10 years ago and achieved significant weight loss. He visited our outpatient clinic for epigastric pain that had persisted for the past 6 months. He also had an episode of melena which occurred 1 month before presentation. On arrival, his physical examination was unremarkable except for mild tenderness on palpation in the epigastric area. An esophagogastroduodenoscopy (EGD) revealed part of the gastroplasty mesh eroding into the stomach ( $\bullet$ Fig. 1 ), and abdominal computed tomography revealed interruption of the mesh with intraluminal protrusion ( Fig. 2). Laparoscopy was performed, which confirmed the diagnosis of VBG with mesh erosion. The mesh was removed and the VBG was converted to a Roux-en-Y gastric bypass. Follow-up after 8 months showed the postoperative course to have been uneventful.

VBG was introduced by Mason in 1982 [1]. The procedure involved the placement of Marlex mesh or a Silastic ring to reinforce the outlet from the gastric pouch, and erosion of the mesh or band has been reported as one of the complications of the procedure. The average interval to mesh erosion is about 3-4 years after VBG, but it can occur more than 10 years after the operation [2]. Common symptoms include abdominal pain, vomiting, and dysphagia. Although successful endo- scopic treatment of mesh erosion has been reported [2], surgical intervention is still the first choice. VBG is no longer recommended in the treatment of morbid obesity because of poor weight loss and high rates of revisional surgery [3]. However, mesh erosion should always be considered as a possible complication in symptomatic patients who have undergone VBG.

Endoscopy_UCTN_Code_CCL_1AB_2AD_3AF

Competing interests: None

\section{Chi-Ming Tai ${ }^{1,2}$, I-Chang Lin ${ }^{3}$, Chih-Kun Huang ${ }^{2}$}

${ }^{1}$ Department of Internal Medicine, E-Da Hospital, I-Shou University, Kaohsiung, Taiwan, ROC

${ }^{2}$ Bariatric and Metabolic International (BMI) Surgery Center, E-Da Hospital, I-Shou University, Kaohsiung, Taiwan, ROC

${ }^{3}$ Department of Radiology, E-Da Hospital, I-Shou University, Kaohsiung, Taiwan, ROC

\section{References}

1 Mason EE. Vertical banded gastroplasty for obesity. Arch Surg 1982; 117: 701 - 706

2 Adam LA, Silva RGJr, Rizk M et al. Endoscopic argon plasma coagulation of Marlex mesh erosion after vertical-banded gastroplasty. Gastrointest Endosc 2007; 65: 337 - 340

3 Schouten R, Wiryasaputra DC, van Dielen FM et al. Long-term results of bariatric restrictive procedures: a prospective study. Obes Surg 2010; 20: 1617-1626

Bibliography

DOI http://dx.doi.org/

10.1055/s-0033-1344871

Endoscopy 2014; 46: E90

(c) Georg Thieme Verlag KG

Stuttgart · New York

ISSN 0013-726X

\section{Corresponding author}

\section{Chih-Kun Huang, MD}

Bariatric and Metabolic International (BMI)

Surgery Center

E-Da Hospital

1, E-Da Road

Jiau-Shu Village

Yan-Chau District

Kaohsiung 824

Taiwan, ROC

dr.ckhuang@hotmail.com 